Check for updates

Cite this: RSC Adv., 2020, 10, 38244

Received 27th July 2020

Accepted 3rd October 2020

DOI: $10.1039 / \mathrm{dOra06495e}$

rsc.li/rsc-advances

\section{Dual-colour (near-infrared/visible) emitting annexin $V$ for fluorescence imaging of tumour cell apoptosis in vitro and in vivo $†$}

\begin{abstract}
Setsuko Tsuboi ${ }^{\mathrm{a}}$ and Takashi Jin (D) *ab
Indocyanine green (ICG) labelled recombinant annexin $\vee$ proteins (ICG-EGFP-Annexin $\vee$ and ICGmPlum-Annexin $V$ ) were synthesized for dual-colour fluorescence imaging of tumour cell apoptosis in vitro and in vivo. The ICG-labelled fluorescent annexin $\vee$ proteins showed dual (near-infrared and visible) fluorescence emissions with binding ability to phosphatidylserines on the plasma membranes of apoptotic cells. Although several types of fluorescence labelled annexin V (e.g. FITC-annexin V, Cy3and Cy5-annexin V) have been reported, there are no dual-colour (near-infrared/visible) emitting apoptosis-detection probes which can be used in vitro and in vivo. In this paper, the utilities of the dualcolour fluorescent annexin $\mathrm{V}$ are demonstrated for in vitro and in vivo fluorescence imaging of the apoptosis of human breast tumour cells induced by an antibody-drug conjugate, Kadcyla. The results suggest that the present annexin $V$ probes will be useful to visualize the action of anti-cancer drugs against tumours both at the cellular and whole-body level.
\end{abstract}

\section{Introduction}

Recent development of antibody-drug conjugates (ADCs) enables specific binding of anti-cancer drugs to tumour cells for apoptosis-based cancer therapy. ${ }^{1}$ In vivo visualization of tumour apoptosis induced by ADCs is crucial for the evaluation of action of the drugs in cancer therapy and for the development of cancer therapeutics. ${ }^{2}$ For in vivo imaging of ADC-induced apoptosis, we can use several imaging modalities such as single photon emission computed tomography (SPECT), positron emission tomography (PET), and magnetic resonance imaging (MRI). ${ }^{3-7}$ SPECT and PET need to use radioactive tracers for in vivo imaging, where their spatial resolution is not enough to visualize apoptotic cells..$^{5,8}$ Although MRI enables in vivo three-dimensional imaging with a high spatial resolution (0.1-1 mm), the detection sensitivity of MRI is relatively low. ${ }^{4} \mathrm{An}$ alternative of the imaging modality for in vivo detection of apoptotic cells is optical imaging. ${ }^{4,8}$ Compared to SPECT, PET and MRI, optical imaging has a high tempo-spatial resolution $\left(\mathrm{ms} \mu \mathrm{m}^{-1}\right) .^{8}$ Herein, we present non-invasive optical imaging of ADC-induced apoptosis of breast tumour cells in vitro and in vivo using near-infrared (NIR) and visible (VIS) emitting fluorescent annexin $\mathrm{V}$.

${ }^{a}$ RIKEN Center for Biosystems Dynamics Research (BDR), RIKEN Furuedai 6-2-3, Suita, Osaka 565-0874, Japan. E-mail: tjin@riken.jp

${ }^{b}$ Graduate School of Frontier Biosciences, Osaka University, Yamada-oka 1-3, Suita, Osaka 565-0871, Japan

$\dagger$ Electronic supplementary information (ESI) available: Experimental details and supplementary figures and table. See DOI: 10.1039/d0ra06495e
Apoptosis can be induced in cancer cells through intrinsic and extrinsic pathways without inducing an inflammatory response. ${ }^{9}$ During the early process of apoptosis, morphological changes in the plasma membrane induce the asymmetrical distribution of phosphatidylserine (PS) on the outer monolayer of the membrane. ${ }^{10}$ Annexin $\mathrm{V}$, an endogenous protein with binding ability to PS in the presence of $\mathrm{Ca}^{2+}$ ions, is widely used as an optical probe for the detection of apoptosis. ${ }^{11}$ To date, a variety of apoptosis probes based on annexin $\mathrm{V}$ modified with fluorescence, luminescence, magnetic resonance contrast, and radioisotopes have been developed. ${ }^{12-30}$ Among these probes, fluorescein conjugated annexin V (FITC-Annexin V) ${ }^{9}$ is a most popular probe for the detection of apoptotic cells. FITCAnnexin V has been widely used as a fluorescent probe for the apoptosis detection by fluorescence microscopy and fluorescence-activated cell sorting methods. ${ }^{31}$ However, FITCAnnexin $\mathrm{V}$ is not suitable for in vivo imaging of apoptotic cells due to its VIS fluorescence, which is strongly absorbed and scattered by tissues and organs.

Although a few types of NIR-fluorescence labelled annexin V (e.g. Cy5-annexin $\mathrm{V}^{29,30}$ and $800 \mathrm{CW}$-annexin $\mathrm{V}^{27}$ ) have been reported, there are no NIR and VIS emitting dual-colour probes which can be used for the detection of apoptosis both at the cellular (in vitro) and whole-body level (in vivo). In this work, we developed indocyanine green (ICG) labelled recombinant fluorescent annexin $\mathrm{V}^{32}$ probes (ICG-EGFP ${ }^{33}$-Annexin $\mathrm{V}$ and ICG-mPlum ${ }^{34}$-Annexin $\mathrm{V}$, Scheme 1) that enable both in vitro and in vivo fluorescence imaging of breast-tumour cell apoptosis induced by an ADC, trastuzumab emtansine ${ }^{35}$ ( $\mathrm{T}$ DM1, Kadcyla). Kadcyla is a conjugate between a humanized 


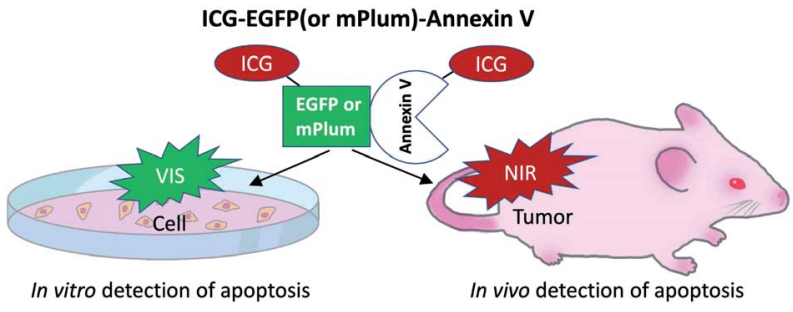

Scheme 1 NIR/VIS emitting dual-colour probes (ICG-EGFPAnnexin $V$ and ICG-mPlum-Annexin $V$ ) for fluorescence imaging of tumour cell apoptosis in vitro and in vivo.

monoclonal anti-HER2 (human epidermal growth factor receptor 2$)^{36}$ antibody and maytansinoid (DM1), a highly potent microtubule polymerization inhibitor ${ }^{35}$ Kadcyla acts as an anti-cancer drug against HER2 positive cancer. ${ }^{37}$ By using the dual-colour (NIR/VIS) emitting annexin V probes, we achieved in vitro and in vivo imaging of apoptosis of HER2-positive breast tumour cells (KPL-4) treated with Kadcyla. Our findings suggest that the present dual-colour (NIR/VIS) emitting annexin $\mathrm{V}$ can be used for the evaluation of the action of ADCs against cancerous tumours both at the cellular and wholebody level.

\section{Results and discussion}

\section{Probe design and characterization}

For in vivo optical imaging of apoptotic cells, NIR probes emitting over $700 \mathrm{~nm}$ are desirable. VIS fluorescence (400 to $700 \mathrm{~nm}$ ) is strongly absorbed and scattered by intrinsic chromophores and organelles in cells. In contrast, NIR fluorescence shows higher permeability and lower scattering in living tissues, leading to clearer deep-tissue images. To prepare NIR apoptosis-imaging probes, we labelled recombinant annexin $\mathrm{V}$ proteins with ICG (Fig. 1a, S1 and S2 $†$ ). Annexin V is a $39.8 \mathrm{kDa}$ molecule with 22 lysine residues, and it is easily labelled with $\mathrm{N}$-succinimidyl ester of ICG (ICG-NHS). The molecular weight of EGFP-Annexin V and mPlum-Annexin V is $66.7 \mathrm{kDa}$ and $65.5 \mathrm{kDa}$, where EGFP and mPlum have 19 and 20 lysine residues, respectively. EGFP and mPlum can also be labelled with ICG-NHS ester (Fig. S3†). From the absorption spectra of ICG-labelled recombinant proteins (Fig. 1b), the number of ICG molecule binding to one recombinant protein molecule was estimated to be 3, 4, and 5 for ICG-Annexin V, ICG-EGFPAnnexin V and ICG-mPlum-Annexin V, respectively.

ICG-Annexin $\mathrm{V}$ shows an emission peak at $820 \mathrm{~nm}$, while ICG-EGFP-Annexin V and ICG-mPlum-Annexin V show NIR/ VIS dual-emission peaks resulting from ICG and fluorescent proteins (Fig. 2a). ICG-EGFP-Annexin $\mathrm{V}$ has emission maxima at $515 \mathrm{~nm}$ (for EGFP) and $820 \mathrm{~nm}$ (for ICG), where the quantum yield of EGFP and ICG emission was estimated to be $35 \%$ and ca.1\% (Table $\mathrm{S} 1 \dagger$ ), respectively. ICG-mPlum-Annexin $\mathrm{V}$ has emission maxima at $640 \mathrm{~nm}$ and $820 \mathrm{~nm}$, where the quantum yield of mPlum and ICG emission was 7\% and $c a .1 \%$ (Table S1†), respectively. To visualize the NIR/VIS dual-emission nature of ICG-EGFP-Annexin V and ICG-mPlum-Annexin V, a)

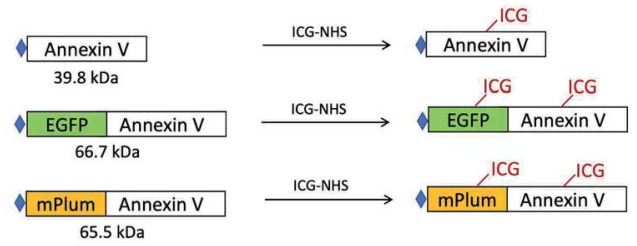

b)
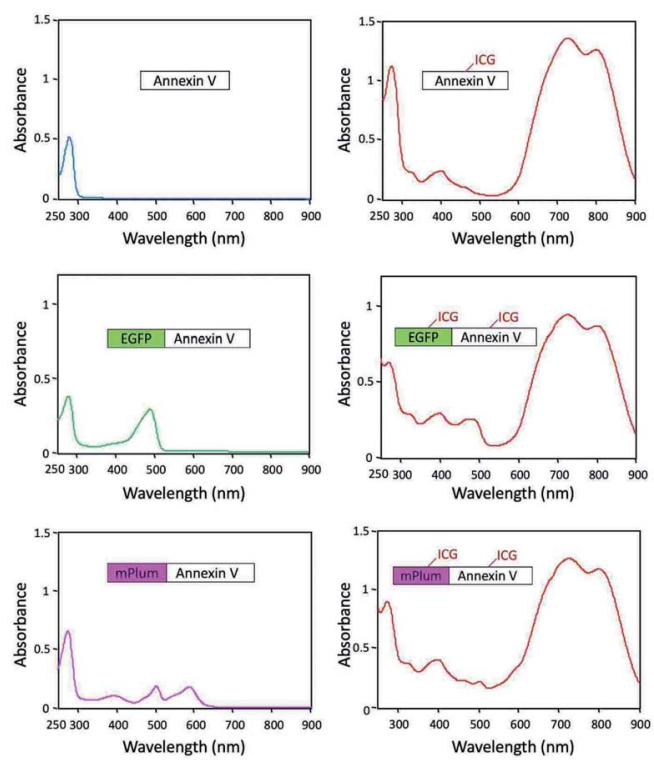

Fig. 1 (a) Fluorescence labelling of Annexin V, EGFP-Annexin $V$, and mPlum-Annexin $V$ with ICG-NHS. The symbol of shows a histidine tag. (b) Absorption spectra of Annexin V, EGFP-Annexin V, and $m$ Plum-Annexin $V$ in phosphate buffered saline (PBS) before and after labelling with ICG-NHS.

we measured their fluorescence images by excitation at $470 \mathrm{~nm}$ for EGFP, $590 \mathrm{~nm}$ for mPlum, and $760 \mathrm{~nm}$ for ICG. ICG-EGFPAnnexin V and ICG-mPlum-Annexin V show green/NIR dual fluorescence and far-red/NIR dual fluorescence, respectively (Fig. 2b). This NIR and VIS dual emission property suggests that ICG-EGFP-Annexin V and ICG-mPlum-Annexin V can be used for the VIS and NIR fluorescence imaging of apoptotic cells both in vitro and in vivo.

Although ICG is a widely used NIR dye for in vivo fluorescence imaging, the drawback of ICG is low photo- and chemicalstabilities in physiological buffer solutions. For instance, ICG degrades in PBS resulting in a loss of absorption and fluorescence because of the formation of reduced non-fluorescence species. ${ }^{38}$ To check the practical usefulness of ICG-EGFPAnnexin V and ICG-mPlum-Annexin V, we examined their photostability in PBS. The time course of fluorescence intensity of ICG-EGFP-Annexin V and ICG-mPlum-Annexin V in PBS showed that the fluorescence emissions of ICG as well as EGFP and mPlum were not drastically changed for one week (Fig. 3). In addition, we observed that the irradiation of excitation lights $\left(3 \mathrm{~mW} \mathrm{~cm}^{-2}\right)$ to the aqueous solutions of ICG-EGFP-Annexin $\mathrm{V}$ and ICG-mPlum-Annexin V did not result in significant photobleaching (Fig. S4†). 

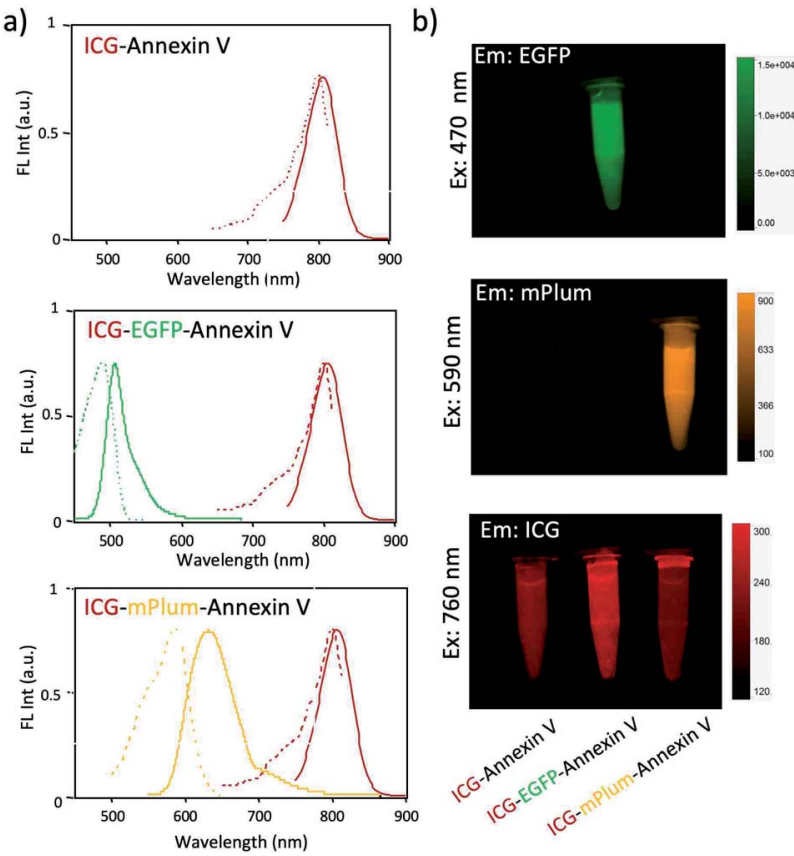

Fig. 2 (a) Fluorescence spectra of ICG-Annexin V, ICG-EGFPAnnexin V, and ICG-mPlum-Annexin V in PBS. Solid and broken lines show their emission and excitation spectra, respectively. (b) Pseudocoloured fluorescence images of ICG-Annexin V, ICG-EGFPAnnexin $V$, and ICG-mPlum-Annexin $V$ in PBS. The fluorescence images of EGFP (em: $515 \pm 20 \mathrm{~nm}$ ), mPlum (em: $670 \pm 20 \mathrm{~nm}$ ), and ICG (em: $830 \pm 20 \mathrm{~nm}$ ) were taken by excitation at 470,590, and $760 \mathrm{~nm}$, respectively.
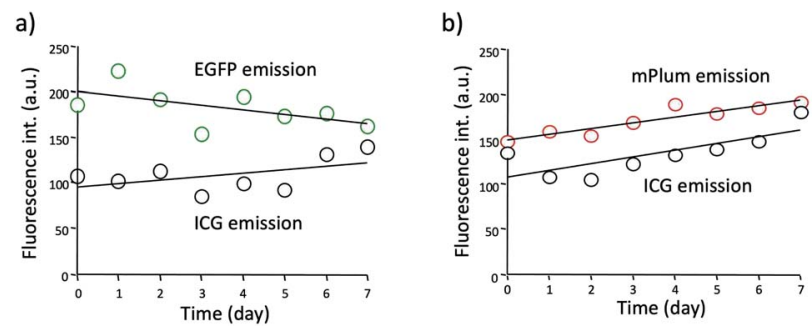

Fig. 3 Time course of fluorescence intensities of ICG, EGFP and mPlum emissions in (a) ICG-EGFP-Annexin $V$ and (b) ICG-mPlumAnnexin $V$ in PBS. Fluorescence intensities are detected at $515 \mathrm{~nm}$ for EGFP, $670 \mathrm{~nm}$ for mPlum, and $830 \mathrm{~nm}$ for ICG. The concentration of probe was $15 \mu \mathrm{M}$.

\section{Fluorescence imaging of apoptotic breast tumour cells in vitro}

For the fluorescence imaging of tumour cell apoptosis, we used a human breast tumour cell line (KPL-4), ${ }^{39}$ which overexpresses HER2 on its cell surface. To observe the apoptosis of KPL-4 cells, we first used a widely used FITC-Annexin V as an apoptosisdetection probe. Apoptosis of KPL4 cells was induced by Kadcyla. After the treatment of KPL-4 cells with Kadcyla for three days, fluorescence imaging of KPL-4 cells was performed by using FITC-Annexin V (Fig. 4a). The KPL-4 cells treated with Kadcyla showed the significant emission of fluorescein (green emission) from the plasma membrane of KPL-4 cells, indicating a) FITC-Annexin V
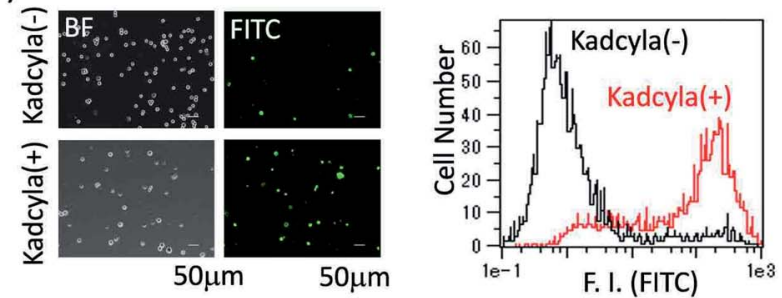

b) ICG-EGFP-Annexin V
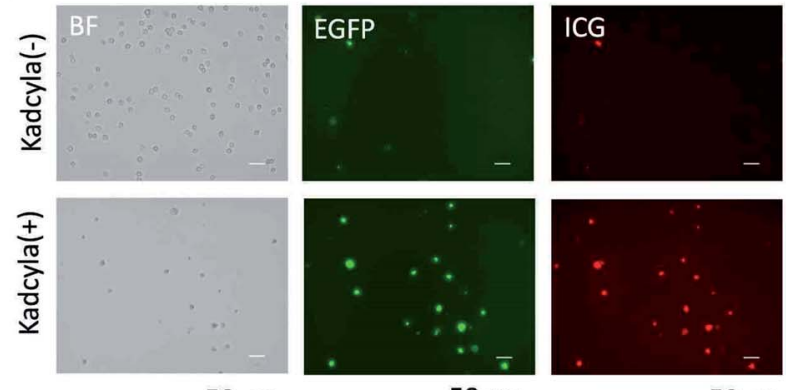

c) ICG-mPlum-Annexin V
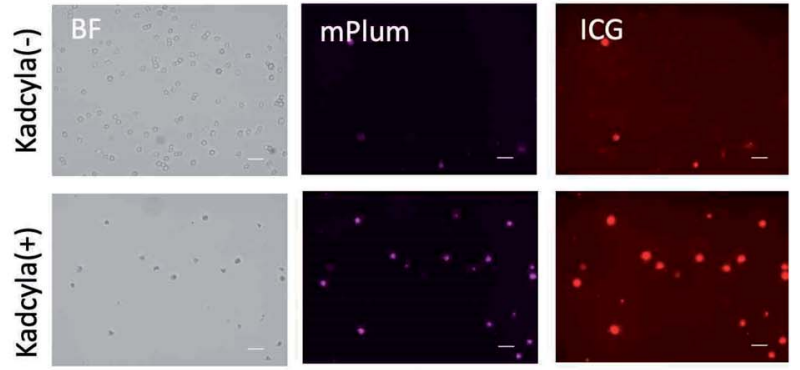

$50 \mu \mathrm{m}$

$50 \mu \mathrm{m}$

$50 \mu \mathrm{m}$

Fig. 4 Bright field (BF) and fluorescence images of KPL-4 cells treated with and without Kadcyla $(10 \mathrm{nM})$. Cellular imaging and flow cytometric analysis were performed three days after the treatment of Kadcyla. (a) KPL-4 cells were stained with FITC-Annexin V. Green colour shows the emission from FITC. The right graph shows the flow cytometric analysis of KPL-4 cells treated with and without Kadcyla. (b) KPL- 4 cells were stained with ICG-EGFP-Annexin $V$. The emissions of EGFP (green) and ICG (red) were detected at $525 \pm 25 \mathrm{~nm}$ (ex: $470 \pm$ $20 \mathrm{~nm}$ ) and $832 \pm 19 \mathrm{~nm}$ (ex: $769 \pm 20 \mathrm{~nm}$ ), respectively. (c) KPL-4 cells were stained with ICG-mPlum-Annexin V. The emissions of mPlum (magenta) and ICG (red) were detected at > $590 \mathrm{~nm}$ (ex: $560 \pm$ $20 \mathrm{~nm}$ ) and $832 \pm 19 \mathrm{~nm}$ (ex: $769 \pm 20 \mathrm{~nm}$ ), respectively.

that the apoptosis of KPL-4 cells was induced by Kadcyla (Fig. 4a and $\mathrm{S} 5 \dagger$ ). In contrast, the control KPL-4 cells (with no Kadcyla) showed only a weak fluorescein emission from the cell membrane Fig. 4a. Flow cytometric analysis showed that the apoptosis of KPL-4 cells was induced by a factor of $76 \%$ by treated with $10 \mathrm{nM}$ of Kadcyla for $72 \mathrm{~h}$ (a right graph in Fig. 4a).

We next checked whether the apoptosis of KPL-4 cells can be detected by the fluorescence of ICG-EGFP-Annexin V and ICGmPlum-Annexin V. In this case, we performed VIS and NIR dual-colour fluorescence imaging of the apoptosis of KPL-4 cells. Fluorescence images of KPL-4 cells by incubated with ICG-EGFP-Annexin V and ICG-mPlum-Annexin V showed the 
VIS and NIR intense emissions from the cells treated with Kadcyla, but not from the cells treated with no Kadcyla (Fig. 4b and c). This result shows that ICG-EGFP-Annexin V and ICGmPlum-Annexin V can be used as VIS/NIR dual-colour fluorescent probes for the detection of apoptosis in vitro. Blocking experiments using unlabelled Annexin V supported the practical usefulness of ICG-EGFP-Annexin V and ICG-mPlumAnnexin $\mathrm{V}$ as fluorescent probes for the detection of apoptotic cells (Fig. S6†).

\section{Breast-tumour bearing mice}

For in vivo imaging of tumour apoptosis, we used breast-tumour bearing mice. Breast-tumour bearing mice were prepared by implanting of KPL-4 cells to nude mice. ${ }^{39 c}$ Tumour apoptosis was induced by an intravenous injection of Kadcyla to the nude mouse. First, we checked whether Kadcyla can accumulate to a HER2-positive breast tumour in the nude mouse. For in vivo fluorescence imaging of Kadcyla, we labelled Kadcyla with ICG, and ICG-labelled Kadcyla was intravenously injected to the mouse via its tail vein. Immediately after the injection of ICGlabelled Kadcyla, fluorescence emission of ICG was observed from the liver (Fig. 5a). Three days after the injection, intense fluorescence emission of ICG was observed from the tumour, indicating the accumulation of Kadcyla to the HER2-positive breast tumour. Ex vivo fluorescence imaging of a breast tumour and organs clearly showed the specific accumulation of Kadcyla to the breast tumour (Fig. 5b).

a)

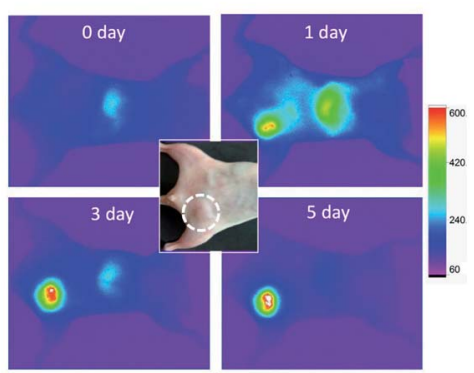

b)

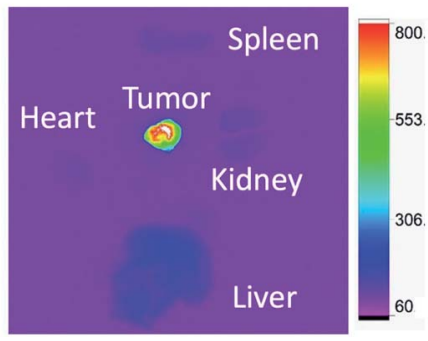

Fig. 5 NIR fluorescence images of a breast-tumour bearing mouse, where an aqueous solution $(200 \mu \mathrm{L})$ of ICG-labelled Kadcyla (1 mg $\mathrm{mL}^{-1}$ ) was intravenously injected via a tail vein of the mouse. A dotted circle in the bright field image of a mouse shows the position of a tumour. (a) NIR fluorescence images of the mouse were taken $0,1,3$, and 5 days after the injection of ICG labelled Kadcyla. (b) Ex vivo image shows NIR fluorescence emission from an isolated breast tumour and organs. NIR fluorescence (em: $830 \pm 20 \mathrm{~nm}$ ) was observed by excitation at $760 \mathrm{~nm}$. Exposure time was $30 \mathrm{~s}$.

\section{Fluorescence imaging of apoptotic breast tumour cells in vivo}

To image tumour apoptosis, we injected ICG-EGFP-Annexin V and ICG-mPlum-Annexin V to the breast tumour-bearing mice treated with Kadcyla for three days (Fig. 6a). Fluorescence imaging of the mice was started three days after the injection of Kadcyla (Fig. 6a). Fluorescence emission of ICG was observed from a breast tumour one day after the injection of ICG-EGFPAnnexin V (Fig. S7 $\dagger$ ). After three days after the injection of the ICG-EGFP (or mPlum)-Annexin V, significant ICG fluorescence from the breast tumour was clearly observed (Kadcyla $(+)$ in Fig. $6 \mathrm{~b}$ and $\mathrm{c}$ ), indicating the accumulation of the Annexin $\mathrm{V}$ probes to the tumour. In the VIS region, EGFP or mPlum emission from the tumour was not clearly observed due to the strong emission of tissue autofluorescence (Fig. S8†). ICGAnnexin $\mathrm{V}$ also accumulated to the breast tumour treated with Kadcyla (Fig. S9†). For the control mice treated with no Kadcyla, we observed only weak fluorescence emissions resulting from the accumulation of ICG-EGFP-Annexin V and ICG-mPlum-

a)

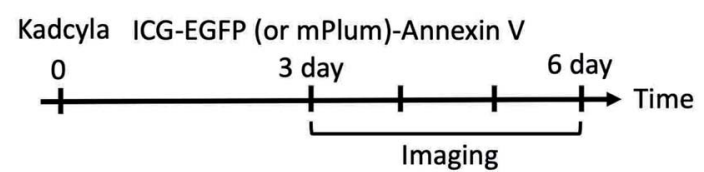

b)
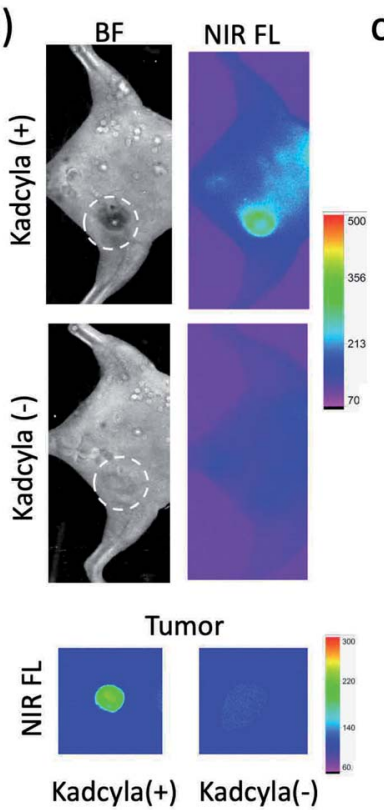
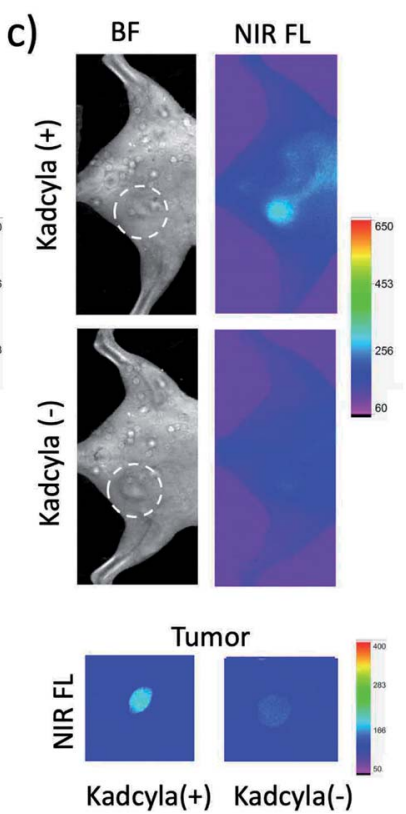

Fig. 6 (a) Time course of experimental procedure for Kadcyla, ICGEGFP (or mPlum)-Annexin $V$ administration and fluorescence imaging. Kadcyla ( $200 \mu \mathrm{L}, 1 \mathrm{mg} \mathrm{mL}^{-1}$ ) was intravenously injected via a tail vein of breast tumour-bearing mice. ICG-EGFP (or Plum)-Annexin $\vee$ was injected to the mice three days after the injection of Kadcyla. (b) Bright filed and NIR fluorescence (ICG-EGFP-Annexin V) images of a mouse treated with and without Kadcyla. (c) Bright filed and NIR fluorescence (ICG-mPlum-Annexin V) images of a mouse treated with and without Kadcyla. Dotted circles show the positions of breast tumours in the mice. Ex vivo images show NIR fluorescence emissions from isolated breast tumours. NIR fluorescence (em: $830 \pm 20 \mathrm{~nm}$ ) was observed by excitation at $760 \mathrm{~nm}$. Exposure time was $30 \mathrm{~s}$. 
Annexin $\mathrm{V}$ to the tumour (Kadcyla $(-)$ in Fig. $6 \mathrm{~b}$ and c). The emission intensity ratio between the breast tumours treated with and without Kadcyla was 6.3 for an ICG-EGFP-Annexin Vinjected mouse and 2.4 for an ICG-mPlum-Annexin V-injected mouse (lower images in Fig. 6b and c).

To confirm the specific accumulation of ICG-EGFPAnnexin V and ICG-mPlum-Annexin V to the apoptotic tumour, we performed a control experiment using ICG labelled EGFP (ICG-EGFP) and ICG-labelled mPlum (ICG-mPlum), which have no annexin $\mathrm{V}$ moieties.

When ICG-EGFP and ICG-mPlum with no annexin V moieties were injected to breast-tumour bearing mice treated with Kadcyla, significant fluorescence emissions were not observed from the tumours (Fig. S10 $\dagger$ ). The control experiment clearly showed that ICG-EGFP-Annexin V and ICG-mPlum-Annexin V accumulated to the tumour by binding of its annexin $\mathrm{V}$ moiety to PS molecules on the plasma membrane of apoptotic tumour cells.

\section{Conclusion}

In this paper, we present ICG labelled recombinant annexin $\mathrm{V}$ probes (ICG-EGFP-Annexin V and ICG-mPlum-Annexin V) for NIR and VIS fluorescence imaging of tumour cell apoptosis. The objective of this work is to develop new fluorescent annexin $\mathrm{V}$ probes which can be used for the detection of apoptosis both at the cellular and whole-body level. Although several types of fluorescent annexin $\mathrm{V}$ have been reported, there are no fluorescent annexin $\mathrm{V}$ probes which can be used for the optical detection of apoptotic cells both in vitro and in vivo. By using the present annexin $\mathrm{V}$ probes, we demonstrate the capability of VIS and NIR dual-colour imaging for the detection of apoptosis. Deregulation in apoptosis induces numerous diseases such as cancer, cardiovascular, and neurodegenerative diseases. We believe that the present VIS/NIR dual-colour emitting annexin V probes will greatly contribute to the study of apoptosis-related diseases at the whole-body level as well as the cellular level.

\section{Experimental}

\section{Materials and instruments}

$N$-Succinimidyl ester derivative of ICG (ICG-NHS) was purchased from Goryo Chemicals (Japan). Kadcyla was purchased from Chugai Pharmaceutical Co., Ltd (Tokyo, Japan). Breast tumour cells were kindly provided by Dr Kurebayashi (Kawasaki Medical School). Nude mice (five-week-old female $\mathrm{BALB} / \mathrm{c} \mathrm{nu} / \mathrm{nu}$ mice) were purchased from Nihon SLC Inc (Japan).

Absorption spectra were recorded with a spectrophotometer (V-670, Jasco). Fluorescence spectra were recorded with a spectrofluorometer (FP-8200, Jasco). Cellular imaging was performed with a fluorescence microscope (BZ-X700, Keyence). Flow cytometric analysis was performed using MACSQuant analyzer (Miltenyi Biotec Inc.) In vivo NIR fluorescence imaging was performed using a fluorescence system (Bruker MS-FX PRO).

\section{Preparation of recombinant proteins (Annexin V, EGFP-} Annexin V and mPlum-Annexin V)

(a) Construction of the recombinant plasmids, pRSETAnnexin V, pRSET-EGFP-Annexin V, and pRSET-mPlumAnnexin V. Annexin V sequence was amplified by PCR from pET12a-PAPI, which was a gift from Jonathan Tait (Addgene plasmid \#19961, B. L. Wood, D. F. Gibson, and J. F. Tait, Blood 1996, 88, 1873-1880). EGFP sequence was amplified by PCR from pEGFP-C1 plasmid (Clontech). mPlum sequence was amplified by PCR from pmPlum vector (Clontech). The PCR fragments were fused with pRSET plasmid (ThermoFisher) by using an InFusion HD cloning kit (Clontech).

(b) Expression of the recombinant proteins in $E$. coli. The pRSET-Annexin V, pRSET-EGFP-Annexin V and pRSETmPlum-Annexin $\mathrm{V}$ plasmids were transformed into $E$. coli $\mathrm{KRX}$ competent cells (Promega). Transformed E. coli were grown as a preculture $(2 \mathrm{~mL})$ in LB medium containing ampicillin $(100 \mu \mathrm{g}$ $\left.\mathrm{mL}^{-1}\right)$ at $37^{\circ} \mathrm{C}$ overnight. For large-scale cultures, the overnight culture $(2 \mathrm{~mL})$ was grown in $200 \mathrm{~mL}$ of $\mathrm{LB}$ medium containing ampicillin $\left(100 \mu \mathrm{g} \mathrm{mL}{ }^{-1}\right)$ at $37^{\circ} \mathrm{C}$, until they approached to 0.6 of OD 600 (absorbance). To induce production of the targeted protein, isopropyl $\beta$-D-1-thiogalactopyranoside $(0.2 \mathrm{mM})$ and $\mathrm{L}^{-}$ rhamnose $(0.1 \%)$ were added to the LB medium, and then incubated with shaking gently for $16 \mathrm{~h}$ at $18{ }^{\circ} \mathrm{C}$.

(c) Extraction and purification of the recombinant proteins. The cells were harvested by centrifugation at $5000 \times g$ for $10 \mathrm{~min}$. The pellet was resuspended in $5 \mathrm{~mL}$ of binding buffer (50 mM Tris- $\mathrm{HCl}, 500 \mathrm{mM} \mathrm{NaCl}, 20 \mathrm{mM}$ imidazole, $\mathrm{pH} 8.0$ ). Before cell lysis, complete EDTA-free protease inhibitor cocktail tablets $(1 \times$, Roche) were added as a protease inhibitor. The suspension was sonicated on ice. Bursts of 10 seconds with intermediate intensity are repeated 7-10 times with a 10 second cooling period between each burst. The lysate was clarified by centrifugation at $20000 \times g$ for 30 min to eliminate cell debris. The supernatant was then purified by Ni Sepharose 6 Fast Flow (GE Healthcare). $0.5 \mathrm{~mL}$ of Sepharose media equilibrated with binding buffer was added to each $5 \mathrm{~mL}$ of lysed sample, and incubated with gentle agitation at $4{ }^{\circ} \mathrm{C}$ for $60 \mathrm{~min}$. After the solution was transferred to an empty column, it was washed with binding buffer five column volumes. Lastly, the recombinant proteins were drained from the column by the addition of elution buffer (50 mM Tris- $\mathrm{HCl}, 500 \mathrm{mM} \mathrm{NaCl}, 500 \mathrm{mM}$ imidazole, $\mathrm{pH}$ 8.0). The eluted fractions were further purified by a size-exclusion column (PD-10, GE Healthcare) to exchange buffer.

\section{ICG labelling of Kadcyla and recombinant proteins}

ICG-NHS ester $(1 \mathrm{mg})$ was resolved to $1 \mathrm{~mL}$ of anhydrous dimethyl sulfoxide. To an aqueous solution (0.01 $\left.\mathrm{M} \mathrm{Na}_{2} \mathrm{CO}_{3}\right)$ of Kadcyla (1 mg mL ${ }^{-1}$ ) or recombinant proteins $\left(1 \mathrm{mg} \mathrm{mL}^{-1}\right), 30$ $\mu \mathrm{L}$ of a dimethyl sulfoxide solution of ICG-NHS was dropwisely added. The coupling reaction was performed for $30 \mathrm{~min}$ at room temperature. The ICG conjugates were purified by a sizeexclusion column (PD-10, GE Healthcare) to remove unreacted dyes. 


\section{SDS PAGE}

The proteins were run on a 5-20\% polyacrylamide gel (Extra PAGE one Precast gel, Nacalai Tesque) in Tris-glycine-SDS buffer, $200 \mathrm{~V}$ for $40 \mathrm{~min}$ and stained with Coomassie Brilliant Blue (CBB Stain One Super, Nacalai Tesque). The sizes of Annexin V, EGFP-Annexin V and mPlum-Annexin V calculated from the amino acid sequence were $39.8 \mathrm{kDa}, 66.7 \mathrm{kDa}$ and 65.5 kDa, respectively. Precision Plus Protein Standard (BIO-RAD) was used as a size marker.

\section{Cellular imaging and flow cytometric analysis}

KPL-4 cells were plated to $35 \mathrm{~mm}$ cell culture dishes $\left(3 \times 10^{5}\right.$ cells per dish) and incubated in Dulbecco's Modified Essential Medium supplemented with $10 \%$ fetal bovine serum, $100 \mu \mathrm{g}$ $\mathrm{mL}^{-1}$ penicillin, and $10 \mu \mathrm{g} \mathrm{mL}^{-1}$ streptomycin in $5 \% \mathrm{CO}_{2}$ at $37{ }^{\circ} \mathrm{C}$ for overnight. Then, KPL-4 was exposed to Kadcyla (10 nM or none) for $72 \mathrm{~h}$. All the cells floating in the medium and the cells that had detached during the PBS washing were collected, and the cells attached to the dish were carefully detached by trypsinization and added to the cell suspension. The cells were washed once with PBS and then resuspended in $1 \mathrm{~mL}$ of Annexin V binding buffer (Nacalai Tesque).

The cell suspension was divided into $100 \mu \mathrm{L}$ aliquots, added with FITC-Annexin V ( $5 \mu$ l; FITC-Annexin V Apoptosis Detection Kit, Nacalai Tesque), ICG-EGFP-Annexin V (final concentration, $0.7 \mu \mathrm{M}$ ) or ICG-mPlum-Annexin V (final concentration, $0.7 \mu \mathrm{M}$ ) and incubated at room temperature for 15 minutes in the dark. As a blocking control, cell suspensions were preincubated with unlabeled recombinant Annexin V (final concentration $4 \mu \mathrm{M}$ ) for 15 minutes at room temperature prior to this staining step. Then, $400 \mu \mathrm{L}$ of binding buffer was added to the stained cell suspension, which was passed through a $35 \mu \mathrm{m}$ cell strainer before use in the next experiment. Quantification by flow cytometry was performed using the MACSQuant Analyzer (Miltenyi Biotec Inc.). FITC-Annexin V was collected through a FL2 filter (ex: $488 \mathrm{~nm}$, em: $525 \pm 25 \mathrm{~nm}$ ). Fluorescence images were acquired with a fluorescence microscope (BZ-X700, Keyence Corp., Japan). The filter set for FITC and EGFP was ex: $470 \pm$ $20 \mathrm{~nm}$, em: $525 \pm 25 \mathrm{~nm}$. The filter set for mPlum was ex: $560 \pm$ $20 \mathrm{~nm}$, em: $590 \mathrm{~nm}$ LP. The filter set for ICG was ex: $769 \pm$ $20 \mathrm{~nm}$, em: $832 \pm 19 \mathrm{~nm}$.

\section{Preparation of tumour-bearing mice}

A suspension of KPL- 4 cells ( $\sim 10^{7}$ cells per mouse) was transplanted to the ventral side of 5 week old female BALB/c nu/nu mice. After several weeks, we selected a mouse bearing a tumour less than $10 \mathrm{~mm}$ in diameter for imaging.

\section{In vivo fluorescence imaging of tumour-bearing mice}

For the fluorescence imaging of a breast-tumour, an aqueous solution $(200 \mu \mathrm{L})$ of ICG-labelled Kadcyla $\left(1 \mathrm{mg} \mathrm{mL} \mathrm{mL}^{-1}\right)$ was intravenously injected via a tail vein of the mouse. NIR fluorescence images of the tumour were taken $0,1,3$, and 5 days after the injection of ICG labelled Kadcyla. Five days after of the probe, ex vivo images of a breast tumour and organs were taken.
For the fluorescence imaging of tumour-apoptosis, Kadcyla $\left(200 \mu \mathrm{L}, 1 \mathrm{mg} \mathrm{mL}^{-1}\right)$ was intravenously injected via a tail vein of breast tumour-bearing mice. Three days after the injection of Kadcyla, ICG-EGFP (or Plum)-Annexin V or ICG-Annexin V (200 $\mu \mathrm{L}, 1 \mathrm{mg} \mathrm{mL}^{-1}$ ) was injected to the mice. Three days after the probes, fluorescence images of a mouse treated were taken.

Fluorescence images were taken using an in vivo fluorescence imaging system (Bruker, MS FX PRO). NIR fluorescence of ICG was observed at $830 \pm 20 \mathrm{~nm}$ by excitation at $760 \mathrm{~nm}$. Exposure time of the excitation light was $30 \mathrm{~s}$. VIS fluorescence of EGFP and mPlum was observed at $515 \pm 20 \mathrm{~nm}$ (ex: $470 \mathrm{~nm}$ ) and $670 \pm 20 \mathrm{~nm}$ (ex: $590 \mathrm{~nm}$ ), respectively. Exposure time of the excitation light was $1 \mathrm{~s}$ for EGFP emission and $10 \mathrm{~s}$ for mPlum emission. Excitation light (400 W Xenon lamp) power was $30 \mu \mathrm{W} \mathrm{cm}{ }^{-2}$ at a ventral side of a mouse.

\section{Ethical statement}

Mice maintenance and animal experiments were performed in accordance with the Guidelines for Care and Use of Laboratory Animals of RIKEN and approved by the Animal Ethics Committee of RIKEN.

\section{Conflicts of interest}

There are no conflicts to declare.

\section{Acknowledgements}

The authors thank Sayumi Yamada and Satoko Masa for their help with animal experiments and manuscript preparations. This work is partly supported by the Ministry of Education, Science, Sport, and Culture of Japan (Grant-in-Aid for Scientific Research (B): $19 \mathrm{H} 04459$ to TJ).

\section{Notes and references}

1 (a) C. H. Chau, P. S. Steeg and W. D. Figg, Lancet, 2019, 394, 793-804; (b) J. M. Lambert and A. Berkenblit, Annu. Rev. Med., 2018, 69, 191-207.

2 S. Kim, K. Cha and I. S. Kim, Curr. Mol. Imaging, 2012, 1, 5562.

3 X. Jiang, E. T. McKinley, J. Xie, H. Li, J. Xu and J. C. Gore, Sci. Rep., 2019, 9, 9540.

4 W. Zeng, X. Wang, P. Xu, G. Liu, H. S. Eden and X. Chen, Theranotics, 2015, 5, 559-582.

5 C. Vangestel, M. Peeters, G. Mees, R. Oltenfreiter, H. H. Boersma, P. H. Elsinga, C. Reutelingsperger, N. V. Damme, B. D. Spiegeleer and C. Van de Wiele, Mol. Imaging, 2011, 10, 340-358.

6 F. G. Blankenberg, J. Nucl. Med., 2008, 49, 81S-95S.

7 F. G. Blankenberg, Cancer Biol. Ther., 2008, 7, 1525-1532.

8 A. R. Kherlopian, T. Song, Q. Duan, M. A. Neimark, M. J. Po, J. K. Gohagan and A. F. Laine, BMC Syst. Biol., 2008, 2, 74.

9 (a) B. A. Carneiro and W. S. El-Deiry, Nat. Rev. Clin. Oncol., 2020, 17, 395-417; (b) R. C. Taylor, S. P. Cullen and 
S. J. Martin, Nat. Rev. Mol. Cell Biol., 2008, 9, 231-241; (c) S. Elmore, Toxicol. Pathol., 2007, 35, 495-516.

10 (a) H. O. van Genderen, H. Kenis, L. Hofstra, J. Narula and C. P. M. Reutelingsperger, Biochim. Biophys. Acta, 2008, 1783, 953-963; (b) M. A. Lizarbe, J. L. Barrasa, N. Olmo, F. Gavilanes and J. Turnay, Int. J. Mol. Sci., 2013, 14, 26522683.

11 M. Nazari, A. Minai-Tehrani and R. Emamzadeh, RSC Adv., 2014, 4, 45128-45135.

12 L. Ruan, M. Ge, X. Huang and J. Ren, Langmuir, 2018, 34, 10040-10047.

13 P. M. S. D. Cal, F. Sieglitz, F. M. F. Santos, C. P. Carvalho, A. Guerreiro, J. B. Bertoldo, U. Pischel, P. M. P. Gois and G. J. L. Bernardes, Chem. Commun., 2017, 53, 368-371.

14 S. Tsuboi and T. Jin, ChemBioChem, 2017, 18, 2231-2235.

15 T. Head, P. Dau, S. Duffort, P. Daftarian, P. M. Joshi, R. Vazquez-Padron, S. K. Deo and S. Daunert, Cell Death Dis., 2017, 8, e2826.

16 T. Zeng, T. Zhang, W. Wei, Z. Li, D. Wu, L. Wang, J. Guo, X. He and N. Ma, ACS Appl. Mater. Interfaces, 2015, 7, 11849-11856.

17 H. K. Jung, K. Wang, M. K. Jung, I. S. Kim and B. H. Lee, PLoS One, 2014, 9, e100341.

18 B. A. Smith, S. T. Gammon, S. Xiao, W. Wang, S. Chapman, R. McDermott, M. A. Suckow, J. R. Johnson, D. PiwnicaWorms, G. W. Gokel, B. D. Smith and W. M. Leevy, Mol. Pharm., 2011, 8, 583-590.

19 X. Huang, S. Lee and X. Chen, Am. J. Nucl. Med. Mol. Imaging, 2011, 1, 3-17.

20 J. Hickson, S. Ackler, D. Klaubert, J. Bouska, P. Ellis, K. Foster, O. Oleksijew, L. Rodriguez, S. Schlessinger, B. Wang and D. Frost, Cell Death Differ., 2010, 17, 1003-1010.

21 G. Niu and X. Chen, J. Nucl. Med., 2010, 51, 1659-1662.

22 K. Wang, S. Purushotham, J. Y. Lee, M. H. Na, H. Park, S. J. Oh, R. W. Park, J. Y. Park, E. Lee, B. C. Cho, M. N. Song, M. C. Baek, W. Kwak, J. Yoo, A. S. Hoffman, Y. K. Oh, I. S. Kim and B. H. Lee, J. Controlled Release, 2010, 148, 283-291.

23 L. Zhao, P. Cheng, J. Li, Y. Zhang, M. Gu, J. Liu, J. Zhang and J. J. Zhu, Anal. Chem., 2009, 81, 7075-7080.

24 L. E. Edgington, A. B. Berger, G. Blum, V. E. Albrow, M. G. Paulick, N. Lineberry and M. Bogyo, Nat. Med., 2009, 15, 967-973.

25 L. Quinti, R. Weissleder and C. H. Tung, Nano Lett., 2006, 6, 488-490.

26 S. Le. Gac, I. Vermes and A. van den Berg, Nano Lett., 2006, 6, 1863-1869.

27 S. Ohnishi, J. L. Vanderheyden, E. Tanaka, B. Patel, A. M. De Grand, R. G. Laurence, K. Yamashita and J. V. Frangioni, Am. J. Transplant., 2006, 6, 2321-2331.

28 S. M. Messerli, S. Prabhakar, Y. Tang, K. Shah, M. L. Cortes, V. Murthy, R. Weissleder, X. O. Breakefield and C. H. Tung, Neoplasia, 2004, 6, 95-105.

29 A. Petrovsky, E. Schellenberger, L. Josephson, R. Weissledser and A. Bogdanov Jr, Cancer Res., 2003, 63, 1936-1942.
30 E. A. Schellenberger, A. Bogdanov Jr, A. Petrovsky, V. Ntziachristos, R. Weissleder and L. Josephson, Neoplasia, 2003, 5, 187-192.

31 (a) B. L. Wood, D. F. Gibson and J. F. Tait, Blood, 1996, 88, 1873-1880; (b) A. W. Boersma, K. Nooter, R. G. Oostrum and G. Stoter, Cytometry, 1996, 24, 123-130; (c) I. Vermes, C. Haanen, H. Steffens-Nakken and C. A. Reutelingsperger, Immunol. Methods, 1995, 184, 39-51; (d) G. Koopman, C. P. Reutelingsperger, G. A. Kuijten, R. M. Keehnen, S. T. Pals and M. H. van Oers, Blood, 1994, 84, 1415-1420.

32 (a) A. Q. Abbady, A. Twair, B. Ali and H. Murad, Front. Physiol., 2017, 8, 317; (b) M. Stöcker, A. Pardo, C. Hetzel, C. Reutelingsperger, R. Fischer and S. Barth, Protein Expression Purif., 2008, 58, 325-331; (c) J. D. Ernst, L. Yang, J. L. Rosales and V. C. Broaddus, Anal. Biochem., 1998, 260, 18-23.

33 (a) J. A. J. Arpino, P. J. Rizkallah and D. D. Jones, PLoS One, 2012, 7, e47132; (b) R. Y. Tsien, Annu. Rev. Biochem., 1998, 67, 509-544; (c) G. H. Patterson, S. M. Knobel, W. D. Sharif, S. R. Kain and D. W. Piston, Biophys. J., 1997, 73, 2782-2790; (d) G. Zhang, V. Gurtu and S. R. Kain, Biochem. Biophys. Res. Commun., 1996, 227, 707-711.

34 (a) M. M. Moore, S. K. Oteng-Pabi, A. T. Pandelieva, S. L. Mayo and R. A. Chica, PLoS One, 2012, 7, e52463; (b) E. M. Merzlyak, J. Goedhart, D. Shcherbo, M. E. Bulina, A. S. Shcheglov, A. F. Fradkov, A. Gaintzeva, K. A. Lukyanov, S. Lukyanov, T. W. J. Gadella and D. M. Chudakov, Nat. Methods, 2007, 4, 555-557; (c) L. Wang, W. C. Jackson, P. A. Steinbach and R. Y. Tsien, Proc. Natl. Acad. Sci. U. S. A., 2004, 101, 16745-16749.

35 N. Iqbal and N. Iqbal, Mol. Biol. Int., 2014, 2014, 852748.

36 J. M. Lambert and R. V. J. Chari, J. Med. Chem., 2014, 57, 6949-6964.

37 (a) P. Liu, J. Fan, Z. Wang, W. Zai, P. Song, Y. Li and D. Ju, $A M B$ Express, 2020, 10, 107; (b) O. Morimura, T. Minami, T. Kijima, S. Koyama, T. Otsuka, Y. Kinehara, A. Osa, M. Higashiguchi, K. Miyake, I. Nagatomo, H. Hirata, K. Iwahori, T. Takimoto, Y. Takeda, H. Kida and A. Kumanogoh, Biochem. Biophys. Res. Commun., 2017, 488, 596-602; (c) R. Nicoletti, S. Lopez, S. Bellone, E. Cocco, C. L. Schwab, J. D. Black, F. Centritto, L. Zhu, E. Bonazzoli, N. Buza, P. Hui, D. Mezzanzanica, S. Canevari, P. E. Schwartz, T. J. Rutherford and A. D. Santin, Clin. Exp. Metastasis, 2015, 32, 29-38.

38 (a) T. Jin, S. Tsuboi, A. Komatsuzaki, Y. Immaura, Y. Muranaka, T. Sakata and H. Yasuda, MedChemComm, 2016, 7, 623-631; (b) W. Holzer, M. Mauerer, A. Penzkofer, R.-M. Szeimies, C. Abels, M. Landthaler and W. Bäumler, J. Photochem. Photobiol., B, 1998, 47, 155-164.

39 (a) J. Kurebayashi, T. Otsuki, C. K. Tang, M. Kurosumi, S. Yamamoto, K. Tanaka, M. Mochizuki, H. Nakamura and H. Sonoo, Br. J. Cancer, 1999, 79, 707-717; (b) S. Tsuboi, A. Sasaki, T. Sakata, H. Yasuda and T. Jin, Chem. Commun., 2017, 53, 9450-9453; (c) S. Tsuboi and T. Jin, RSC Adv., 2019, 9, 34964-34971. 